TRANSACTIONS OF THE

AMERICAN MATHEMATICAL SOCIETY

Volume 351, Number 9, Pages 3549-3567

S 0002-9947(99)02193-5

Article electronically published on February 5, 1999

\title{
CONVEX FUNCTIONS ON ALEXANDROV SURFACES
}

\author{
YUKIHIRO MASHIKO
}

\begin{abstract}
We investigate the topological structure of Alexandrov surfaces of curvature bounded below which possess convex functions. We do not assume the continuities of these functions. Nevertheless, if the convex functions satisfy a condition of local nonconstancy, then the topological structures of Alexandrov surfaces and the level sets configurations of these functions in question are determined.
\end{abstract}

\section{INTRODUCTION}

A real valued function $\varphi: M \longrightarrow R$ on a complete Riemannian manifold $M$ is by definition convex if it is a geodesically convex function. That is, if $\gamma:[a, b] \longrightarrow M$ is a unit speed geodesic and if $t \in[0,1]$, then

$$
\varphi \circ \gamma((1-t) a+t b) \leq(1-t) \varphi \circ \gamma(a)+t \varphi \circ \gamma(b) .
$$

Typical examples of convex functions on Riemannian manifolds are Busemann functions on complete Riemannian manifolds of nonnegative sectional curvature. Using filtration by compact totally convex sets, J. Cheeger and D. Gromoll investigated the structure theorem by which the topological structure of complete noncompact Riemannian manifolds of nonnegative sectional curvature is determined $([5])$.

It is well known that the existence of a locally nonconstant convex function on a complete Riemannian manifold $M$ imposes strong restrictions on the topology of $M$ ([6], Theorems C, D and F). Here, the local nonconstancy of a convex function means that it is not constant on any open set. As is explained on p.130 of [6], every noncompact smooth manifold $M$ admits a complete Riemannian metric $g$ and a nontrivial smooth function $\varphi$ such that $\varphi$ is convex with respect to $g$. For such a $\varphi$, the minimum set of $\varphi$ has a nonempty interior in which all the topological information of $M$ is included. Thus it is natural to assume that a convex function is locally nonconstant. Geodesic completeness on $M$ plays an essential role in the above results. In fact, the property of local Lipschitz continuity of a convex function on $M$ is derived from geodesic completeness, and also the noncompactness of $M$.

It is natural to ask if certain topological restrictions can be obtained for geodesic spaces admitting locally nonconstant convex functions. In fact, N. Innami proved in Theorem 3.13 of [9] that if a Busemann G-surface admits a locally nonconstant convex function, then it is homeomorphic to either a plane $R^{2}$, a cylinder $S^{1} \times R^{1}$, or an open Möbius strip. Here the geodesic completeness holds on Busemann Gsurfaces.

Received by the editors April 10, 1997.

1991 Mathematics Subject Classification. Primary 53C20.

Key words and phrases. Alexandrov spaces of curvature bounded below, convex functions.

(C)1999 American Mathematical Society 
It is interesting to study convex functions on a connected and complete $n$ dimensional Alexandrov space $X$ without boundary, whose curvature is bounded below. A curve in such an $X$ is by definition geodesic if it possesses the locally minimizing property. We define a convex function on $X$ as done on complete Riemannian manifolds. If $X$ is a noncompact Alexandrov space with nonnegative curvature in the Alexandrov sense, then every Busemann function becomes convex. Therefore one expects a result in this case similar to that obtained in the case of noncompact complete Riemannian manifolds with nonnegative curvature. G. Perelman has investigated the structure of nonnegatively curved Alexandrov spaces (see [12]).

As discussed in [3] $\S 4, X$ admits a singular set, $S_{X}$, and the Hausdorff dimension of $S_{X}$ is at most $\operatorname{dim} X-1$ (see [11], Theorem A). Moreover, geodesic completeness does not hold on $X$, i.e., a geodesic cannot be extended to both its sides. Therefore a convex function $\varphi: X \longrightarrow R$ on $X$ is not necessarily continuous. The $a$-sublevel set $X^{a}$ of $\varphi$ is denoted by

$$
X^{a}:=\{x \in X \mid \varphi(x) \leq a\}, a \in \varphi(X) .
$$

Clearly $X^{a}$ is totally convex. However, it is not necessarily true that the closure of $X^{a}, \overline{X^{a}}$, is totally convex. Here, the discontinuity of $\varphi$ causes serious difficulties. Also, the sublevel set $X^{a}$ of $\varphi$ is not necessarily closed.

We restrict our consideration to the case in which $\operatorname{dim} X=2$. From this point let $X$ be a connected, complete 2-dimensional Alexandrov space without boundary, whose curvature is bounded below by $k$. Such an $X$ is called an Alexandrov surface. Then $S_{X}$ is a countable set (see [10], Lemma 1.3). As stated in the previous paragraph, we do not know a priori that $\sup _{X} \varphi=+\infty$ for a nontrivial convex function $\varphi$. For the discussion of this problem we introduce the notion of the closure supremum of a convex function $\varphi$ :

$$
\sup _{c l} \varphi:=\inf \left\{a \in \varphi(X) \mid \overline{X^{a}}=X\right\} .
$$

In the example due to Otsu and Shioya (stated in $§ 2$ ), every locally nonconstant convex function on it has the property

$$
\sup _{c l} \varphi=\inf _{X} \varphi .
$$

We characterize such surfaces in $\S 3$ as follows.

Theorem A. If a compact Alexandrov surface $X$ possesses a convex function $\varphi$, then (*) is satisfied. Moreover, a compact Alexandrov surface $X$ admits a locally nonconstant convex function if and only if the set $\hat{S}_{X}$ is everywhere dense, where $\hat{S}_{X}:=\{x \in X \mid$ there exists no geodesic which contains $x$ in its interior $\}$.

$\hat{S}_{X}$ includes the singular set $S_{X}$. The author does not know whether there exists an Alexandrov surface $X$ such that there exists an open set $U$ in $X$ in which $S_{X}$ is empty and in which $\hat{S}_{X}$ is dense.

The following proposition play an important role throughout our investigation (see §3). However, it is not certain that Proposition 3.4 (3) is valid for $\operatorname{dim} X \geq 3$.

Proposition $3.4(3)$. For every $a \in\left(\inf _{X} \varphi, \infty\right), \overline{X^{a}}$ is closed totally convex with interior points. 
In order to avoid the case of $(*)$, we consider a convex function $\varphi: X \longrightarrow R$ such that

$$
\inf _{X} \varphi<\sup _{c l} \varphi .
$$

The a-level set, $\partial\left(\overline{X^{a}}\right)$, of $\varphi$ is defined by

$$
\partial\left(\overline{X^{a}}\right):=\overline{X^{a}} \backslash \operatorname{int}\left(\overline{X^{a}}\right), a \in\left(\inf _{X} \varphi, \sup _{c l} \varphi\right) .
$$

In $\S 3$ we investigate $a$-level sets lying above the minimum set and obtain the following results.

Theorem B. If $(* *)$ is satisfied for a convex function $\varphi: X \longrightarrow R$, then each component of $\partial\left(\overline{X^{a}}\right)$ for every $a \in\left(\inf _{X} \varphi, \sup _{c l} \varphi\right)$ has the structure of a properly embedded 1-dimensional topological submanifold without boundary, and hence each is homeomorphic to either $S^{1}$ or $R^{1}$.

Theorem C. If $(* *)$ is satisfied for a convex function $\varphi: X \longrightarrow R$, and if $\partial\left(\overline{X^{a}}\right)$ is compact for some $a \in\left(\inf _{X} \varphi, \sup _{c l} \varphi\right)$, then $\partial\left(\overline{X^{b}}\right)$ is compact for all $b \in\left[a, \sup _{c l} \varphi\right)$.

Proposition 3.6. The following statements hold.

1. The a-level set $\partial\left(\overline{X^{a}}\right)$ for every $a \in\left(\inf _{X} \varphi, \sup _{c l} \varphi\right)$ is a rectifiable curve.

2. Assume that a sequence $\left\{\mathcal{C}_{i}:[0,1] \longrightarrow \partial\left(\overline{X^{c_{i}}}\right)\right\}_{i=1}^{\infty}$ of curves in the $c_{i}$-level sets converges pointwise to a curve $\mathcal{C}:[0,1] \longrightarrow \partial\left(\overline{X^{c_{0}}}\right)$ in the $c_{0}$-level set, where $c_{i} \in\left(\inf _{X} \varphi, \sup _{c l} \varphi\right)$ for all $i=0,1, \cdots$. Then

$$
\lim _{i \rightarrow \infty} L\left(\mathcal{C}_{i}\right)=L(\mathcal{C})
$$

where $L(\cdot)$ represents the length of curve.

Making use of the first variation formula, we prove

Theorem D. Under the same assumptions as in Theorem $C$, the length $L\left(\partial\left(\overline{X^{b}}\right)\right)$ of $\partial\left(\overline{X^{b}}\right)$ is continuous and monotone nondecreasing in $b \in\left[a, \sup _{c l} \varphi\right)$.

Corollary to Theorems B, C and D. If a convex function $\varphi: X \longrightarrow R$ satisfies $(* *)$, then the following hold.

1. If $\partial\left(\overline{X^{a}}\right)$ is compact for some $a \in\left(\inf _{X} \varphi, \sup _{c l} \varphi\right)$, then $\partial\left(\overline{X^{b}}\right)$ is compact for all $b \in\left(\inf _{X} \varphi, \sup _{c l} \varphi\right)$.

2. $\sup _{X} \varphi=\sup _{c l} \varphi=+\infty$.

3. $X$ is noncompact.

In $\S 3$ we prove Theorems B, C, D and A in this order. For the above results we need not assume the local nonconstancy of $\varphi$, because $(* *)$ does not imply that the minimum set has nonempty interior points.

We investigate the complete information on the level sets $\left\{\partial\left(\overline{X^{a}}\right)\right\}_{a \in \varphi(X)}$ of $\varphi$. In this case, the local nonconstancy of $\varphi$ is required. For the purpose of this investigation, we introduce the notion of the $a$-lower ( $a$-upper, resp.) level set $\operatorname{Low}(a)(U p p(a)$, resp.) as follows:

$$
\operatorname{Low}(a):=\operatorname{int}\left(\overline{X^{a}}\right), \operatorname{Upp}(a):=\operatorname{ext}\left(X^{a}\right)\left(=\operatorname{int}\left(\left(X^{a}\right)^{C}\right)=\left(\overline{X^{a}}\right)^{C}\right), a \in \varphi(X) .
$$

Clearly, $X$ is expressed as the disjoint union

$$
X=\operatorname{Low}(a) \cup \partial\left(\overline{X^{a}}\right) \cup U p p(a) .
$$


We may say that the local nonconstancy of (discontinuous) $\varphi$ is equivalent to the property

$$
\operatorname{int}\left(\bigcap_{a>\inf _{X} \varphi} \operatorname{Low}(a)\right)=\emptyset
$$

Clearly, $(* * *)$ implies $(* *)$. Note that all the locally nonconstant convex functions on $X$ do not satisfy $(* * *)$. Then our main theorem is stated as

Main theorem. If a connected, complete Alexandrov surface $X$ of curvature bounded below without boundary possesses a convex function $\varphi: X \longrightarrow R$ satisfying property (***), then $X$ is homeomorphic to either a plane $R^{2}$, a cylinder $S^{1} \times R$ or an open Möbius strip.

In $\S 4$ we obtain detailed information on the level set configuration in $X$. The study of the ends of $X$ is closely related to that of the level set configuration. In $\S 4$ we prove the following results.

Theorem E. If $(* * *)$ is satisfied for a convex function $\varphi: X \longrightarrow R$, then the a-level set $\partial\left(\overline{X^{a}}\right)$ for $a \in\left(\inf _{X} \varphi, \infty\right)$ has at most two components. Moreover, if $\partial\left(\overline{X^{a}}\right)$ for some $a \in\left(\inf _{X} \varphi, \infty\right)$ is disconnected, then

1. $\inf _{X} \varphi=: \lambda$ is attained,

2. the minimum set $X^{\lambda}$ is either a simple closed geodesic or a straight line, and

3. $\partial\left(\overline{X^{b}}\right)$ for all $b \in\left(\inf _{X} \varphi, \infty\right)$ has exactly two components.

Here a straight line is by definition a complete geodesic $\gamma: R \longrightarrow X$ such that $d\left(\gamma\left(t_{1}\right), \gamma\left(t_{2}\right)\right)=\left|t_{1}-t_{2}\right|$ for all $t_{1}, t_{2} \in R$.

Corollary to Theorem E. If (***) is satisfied for a convex function $\varphi: X \longrightarrow R$, then $X$ has at most two ends. Furthermore, $X$ has exactly one end if one of the following two conditions is satisfied:

(1) $\varphi$ has noncompact and connected levels $\left\{\partial\left(\overline{X^{a}}\right)\right\}_{a>\inf _{X} \varphi}$.

(2) $\varphi$ has compact levels $\left\{\partial\left(\overline{X^{a}}\right)\right\}_{a>\inf _{X} \varphi}$ and the minimum of $\varphi$ is attained.

Moreover, $X$ has exactly two ends if one of the following two conditions is satisfied:

(3) $\varphi$ has compact and disconnected levels.

(4) $\varphi$ has compact levels and $\inf _{X} \varphi=-\infty$.

Finally in $\S 4$ we prove Theorem E, its corollary, and our main theorem.

\section{Preliminaries}

Let $M_{\kappa}^{2}$ be a simply connected, complete 2-dimensional Riemannian manifold with constant curvature $\kappa$. Then the axioms for an Alexandrov space $X$ of curvature bounded below by $\kappa$ are the following:

1. $X$ is a locally compact, connected, complete inner metric space.

2. The Hausdorff dimension of $X$ is finite.

3. There exists for every $x \in X$ a neighborhood $U_{x}$ around $x$ satisfying the following property: Let $\triangle=\triangle\left(x_{1}, x_{2}, x_{3}\right)$ be a sufficiently small geodesic triangle contained in $U_{x}$ with vertices $x_{1}, x_{2}, x_{3} \in X$. Draw a corresponding geodesic triangle $\tilde{\triangle}=\triangle\left(\tilde{x}_{1}, \tilde{x}_{2}, \tilde{x}_{3}\right)$ in $M_{\kappa}^{2}$ whose edges are each of the same length as the corresponding edges of $\triangle\left(x_{1}, x_{2}, x_{3}\right)$. Then if we take a point $s$ from the edge $\tilde{x}_{1} \tilde{x}_{2}$ corresponding to a given point $s$ on the edge $x_{1} x_{2}$,

$$
d\left(x_{3}, s\right) \geq d\left(\tilde{x}_{3}, \tilde{s}\right) .
$$


The inequality in the axiom (3) makes it possible to define an angle $\angle_{x}(\gamma, \sigma)$ between two geodesics $\gamma, \sigma$ emanating from $x \in X$. We denote by $\Sigma_{x}^{\prime}$ for each $x \in X$ the set of directions of all geodesics emanating from $x$. We define the space of directions $\Sigma_{x}$ at $x$ by the completion of $\Sigma_{x}^{\prime}$ with respect to the angular metric $\angle_{x}$.

From this point we restrict our discussion to the case that the Hausdorff dimension of $X$ is 2 and $X$ has no boundary. We call such an $X$ an Alexandrov surface of curvature bounded below without boundary, or simply, an Alexandrov surface. Then $\Sigma_{x}$ for every $x \in X$ is a circle of length $\leq 2 \pi$. Denoting by $L\left(\Sigma_{x}\right)$ the length of $\Sigma_{x}$, we call $x \in X$ a singular point (resp. regular point) if $L\left(\Sigma_{x}\right)<2 \pi$ (resp. $\left.L\left(\Sigma_{x}\right)=2 \pi\right)$.

Throughout this paper the following facts are used frequently: Facts 0, 2, 3, 4 and 5 below are valid for higher dimensional Alexandrov spaces of curvature bounded below.

Fact 0. No geodesic branches. That is, if $d(p, s)=d(p, q)+d(q, s), d(p, r)=$ $d(p, q)+d(q, r)$ and if $d(p, r)=d(p, s)$, then $r=s$.

Fact 1 (see [14]). For any $p \in X$ there exists a measure zero set $\Omega \subset(0,+\infty)$ such that $\partial B(p ; t)$ for $t \notin \Omega$ consists of finitely many rectifiable circles. In particular, $B(p ; r)$ for a sufficiently small $0<r \notin \Omega$ is a disk.

Fact 2. Let $p q$ for arbitrary $p, q \in X$ denote a minimal geodesic from $p$ to $q$ and $\angle q p r$ the angle between $p q$ and $p r$ at $p$. If $\lim _{i \rightarrow \infty} p_{i} q_{i}=p q$ and $\lim _{i \rightarrow \infty} p_{i} r_{i}=p r$, then

$$
\liminf _{i \rightarrow \infty} \angle q_{i} p_{i} r_{i} \geq \angle q p r .
$$

Here equality holds if and only if $p$ is a regular point.

Fact 3 (The first variation formula; see [11], Theorem 3.5). For a geodesic $x y$ and for a point $p \in X$ we have

$$
d(p, y)-d(p, x)=-d(x, y) \cdot \cos \left(\min _{p x} \angle p x y\right)+o(d(x, y)),
$$

where the minimum is taken over all geodesics joining $p$ to $x$.

Fact 4 (The slope inequality). Let $\varphi: X \longrightarrow R$ be a convex function on an Alexandrov surface $X$ and $\gamma:[a, b] \longrightarrow X$ a geodesic. Then we have for $s, t, u$ with $a \leq s<t<u \leq b$

$$
\frac{\varphi \circ \gamma(t)-\varphi \circ \gamma(s)}{t-s} \leq \frac{\varphi \circ \gamma(u)-\varphi \circ \gamma(s)}{u-s} \leq \frac{\varphi \circ \gamma(u)-\varphi \circ \gamma(t)}{u-t} .
$$

Fact 5 (see [3]). For an arbitrarily fixed $p \in X$, the pointed Hausdorff limit $\lim _{r \rightarrow 0}\left(\frac{1}{r} X, p\right)$ of the $1 / r$-scaling of $X$ at $p$ becomes a flat cone $\left(K\left(\Sigma_{p}\right), o_{p}\right)$ over $\Sigma_{p}$ with vertex $o_{p}$.

Fact 6 (see Lemma 2.7 in [10]). Assume that for some $x \in X$ there exists a sequence $\left\{\triangle_{i}\right\}$ of geodesic triangles such that $x \in \operatorname{int}\left(\triangle_{i}\right)$ for each $i$ and $\triangle_{i} \rightarrow\{x\}$. Then

$$
\lim _{i \rightarrow \infty} e\left(\triangle_{i}\right)=2 \pi-L\left(\Sigma_{x}\right),
$$

where $e\left(\triangle_{i}\right):=($ the sum of the inner angles of $\triangle)-\pi$. 
For a convex function $\varphi: X \longrightarrow R$ we define

$$
\begin{gathered}
X^{a}(\varphi):=\{x \in X \mid \varphi(x) \leq a\} \text { (sublevel set) } \\
X^{<a}(\varphi):=\{x \in X \mid \varphi(x)<a\} \text { (proper sublevel set) }
\end{gathered}
$$

and

$$
\overline{X_{a}^{b}}(\varphi):=\overline{\operatorname{Low}(b)} \cap \overline{U p p(a)} .
$$

We omit $\varphi$ for convenience.

A subset $C$ of $X$ is by definition a totally convex set if and only if any geodesic joining any points in $C$ is contained entirely in $C$.

We first state the following basic lemma. The proof is elementary and omitted.

Lemma 1.1. Let $\varphi: X \longrightarrow R$ be a convex function. Then the following hold.

1. We have $X^{<a} \subset \operatorname{Low}(a)$ for every $a>\inf _{X} \varphi$.

2. We have $\overline{X^{<a}}=\overline{X^{a}}$ for every $a>\inf _{X} \varphi$, and hence $\operatorname{Low}(a)=\operatorname{int}\left(\overline{X^{<a}}\right)$.

3. Let $p$ be an interior of some geodesic $\gamma:[a, b] \longrightarrow X$; then

(a) If $p \in \operatorname{Low}(a)$ for some $a>\inf _{X} \varphi$, then $\varphi(p)<a$. Moreover, if $p \in \operatorname{Low}\left(\inf _{X} \varphi\right)$, then $\varphi(p)=\inf _{X} \varphi$.

(b) If $p \in U p p(a)$ for some $a \geq \inf _{X} \varphi$, then $\varphi(p)>a$.

(c) If $\varphi(p)=a>\inf _{X} \varphi$, then $p \in \partial\left(\overline{X^{a}}\right)$.

The following lemma follows immediately from Lemma 1.1.

Lemma 1.2. If $\varphi: X \longrightarrow R$ is a convex function, then Low (a) for any $a>\inf _{X} \varphi$ is a totally convex set. Hence $\bigcap_{a>\inf _{X} \varphi} \operatorname{Low}(a)$ is also a totally convex set if it is nonempty. Moreover if $\varphi$ has the property that $\bigcap_{a>\inf _{X} \varphi}$ Low (a) has no interior, then $\bigcap_{a>\inf _{X} \varphi} \operatorname{Low}(a)$ is either a straight line, a ray, a closed geodesic, a geodesic segment, a point, or an empty set.

\section{EXAMPLES}

Example 2.1 (Flat cone). Let $X \subset R^{3}$ be a flat cone over a plane circle $S^{1}$ of length $<2 \pi$ at the origin $o$ of $R^{3}$. Then $X$ is an Alexandrov surface of $\operatorname{curv}(X) \geq 0$ and the tangent cone $\left(K\left(\Sigma_{o}\right), o\right)$ at $o$ is isometric to $X$.

We define a function $\varphi: X \longrightarrow R$ by $\varphi(x):=|x|$ for $x \in X-\{o\}$ and $\varphi(o):=$ $b \geq 0$. Then $\varphi$ is a (locally nonconstant) convex function.

Assume $\varphi(o)=b>0$. Then $\varphi$ is not continuous, and the infinimum of $\varphi$ is not attained at $o$. We see that $\partial\left(\overline{X^{a}}\right)$ for any $a>\inf \varphi$ is a circle $S(o ; a)=$ $\{p \in X|| p \mid=a\}$ and that $\partial X^{a}=\partial\left(\overline{X^{a}}\right) \cup\{o\}$ for any $a \in(0, b)$.

Example 2.2 (Otsu-Shioya; see [11], pp. 632,633). Let $X_{n} \subset R^{3}$ be a compact convex polyhedron with $4 \cdot 3^{n}$-faces and $v_{n}$-vertices such that for every $n=0,1,2, \cdots$ (1) all the vertices of $X_{n}$ are those of $X_{n+1}$, and (2) $v_{n+1}-v_{n}=4 \cdot 3^{n}$. Then the Hausdorff limit $X$ of $\left\{X_{n}\right\}_{n=0,1,2, \ldots}$ is a compact Alexandrov surface with $\operatorname{curv}(X) \geq 0$. The singular set of $X$ is the union of all the vertices of the $X_{n}$ and is dense on $X$ (see [11], pp. 632,633).

Let $\varphi_{1}, \varphi_{2}: X \longrightarrow R$ be

$$
\varphi_{1}(x):= \begin{cases}0 & \text { if } x \text { is regular } \\ 1 & \text { if } x \text { is singular }\end{cases}
$$


and

$$
\varphi_{2}(x):= \begin{cases}0 & \text { if } x \text { is regular, } \\ 2^{n} & \text { if } x \text { is a vertex of } X_{n} \backslash X_{n-1} .\end{cases}
$$

Then $\varphi_{1}$ and $\varphi_{2}$ are locally nonconstant convex and satisfy the equalities $\sup _{X} \varphi_{1}=$ $1, \sup _{X} \varphi_{2}=\infty, \sup c l \varphi_{j}=\inf _{X} \varphi_{j}=0(j=1,2)$, and

$$
\overline{X^{\inf \varphi_{j}}}=\bigcap_{a>\inf \varphi_{j}} \operatorname{Low}(a)=X
$$

(see Theorem A).

\section{LEVEL SETS}

Throughout this section let $\varphi: X \longrightarrow R$ be a convex function satisfying (**).

Let $a$ be a number such that $\inf _{X} \varphi<a<\sup _{c l} \varphi$. Then for every $x, y \in \overline{X^{a}}$ there exists at least one minimal geodesic from $x$ to $y$ contained entirely in $\overline{X^{a}}$. Therefore the following definition makes sense.

Definition 3.1. A subset $T_{x}^{\prime}$ of $\Sigma_{x}^{\prime}$ for $x \in \partial\left(\overline{X^{a}}\right)\left(\inf _{X} \varphi<a<\sup _{c l} \varphi\right)$ is defined as follows: $\xi \in T_{x}^{\prime}$ if and only if $\exp _{x} t \xi \in \overline{X^{a}}$ for some $t>0$ and the geodesic $\gamma_{\xi}:[0, t] \longrightarrow X$ tangent to $\xi$ is contained entirely in $\overline{X^{a}}$. We define

$$
T_{x}:=\overline{T_{x}^{\prime}}
$$

and call it the tangent directions to $\overline{X^{a}}$ at $x$.

$T_{x}$ determines a subarc of $\Sigma_{x}$. We estimate the length $L\left(T_{x}\right)$ of $T_{x}$ as follows and the proof is omitted.

Lemma 3.2. If $x \in X$ is in $\partial\left(\overline{X^{a}}\right)$ for some $a \in\left(\inf _{X} \varphi, \sup _{c l} \varphi\right)$, then

$$
0<L\left(T_{x}\right) \leq L\left(\Sigma_{x}\right)-\pi
$$

Remark 3.3. If there exists a point $x \in X$ such that $L\left(\Sigma_{x}\right) \leq \pi$, then it follows from the previous lemma that $x$ does not belong to $\partial\left(\overline{X^{a}}\right)=\partial \operatorname{Low}(a)=\partial U p p(a)$ for any $a \in\left(\inf _{X} \varphi, \sup _{c l} \varphi\right)$. Therefore we have either

$$
x \in \bigcap_{a>\inf _{X} \varphi} \operatorname{Low}(a) \text { or } \quad x \in \bigcap_{\inf _{X} \varphi<a<\sup _{c l} \varphi} \operatorname{Upp}(a) .
$$

From the corollary to Theorems B, C and D, which will be proved later, we observe that

$$
\bigcap_{\inf _{X} \varphi<a<\sup _{c l} \varphi} U p p(a)=\emptyset
$$

for any convex function on $X$. Thus for a point $x$ such that $L\left(\Sigma_{x}\right) \leq \pi$ we observe

$$
x \in \bigcap_{a>\inf _{X} \varphi} \operatorname{Low}(a) .
$$

Proof of Theorem B. We assert that for every fixed $x \in \partial\left(\overline{X^{a}}\right)$ there is a disk neighborhood $B(x ; r)$ for some sufficiently small $r>0$ such that $B(x ; r) \cap \partial\left(\overline{X^{a}}\right)$ is a Jordan curve. Once this is established, the properness of the $a$-level set is clear. Let $v^{l}$ and $v^{r}$ be two directions which bound the tangent directions $T_{x}$ at $x$ on the right-hand and left-hand sides respectively. We choose a direction $w \notin T_{x}$ such that $\angle\left(v^{r}, w\right), \angle\left(v^{l}, w\right)>\pi / 2-\zeta$ for a sufficiently small $\zeta>0$ and that there exists a 
geodesic $\gamma_{w}$ tangent to $w$ at $x$, i.e., $\dot{\gamma}(0)=w$. Take a point $p$ from the interiors of $\gamma_{w}$ close to $x$ so that $p$ is contained in a disk neighborhood around $x$. Then $p \in U p p(a)$.

There exist two points $x^{r}$ and $x^{l}$ in $\partial\left(\overline{X^{a}}\right)$ sufficiently close to $x$ such that

$$
\angle\left(\left(\dot{x} \dot{x}^{r}\right)(0), v^{r}\right), \quad \angle\left(\left(\dot{x} x^{l}\right)(0), v^{l}\right)<\frac{\zeta}{100},
$$

where $(\cdot)$ represents the initial tangent, and

$$
x x^{r}, x x^{l} \subset B\left(p ; \frac{101}{100} d(p, x)\right) .
$$

We only discuss the case for the right-hand side. The right-hand side of the desired Jordan curve $\lambda_{a}:[0,1] \longrightarrow B(x ; r) \cap \partial\left(\overline{X^{a}}\right)$ is constructed as follows. We put $\lambda_{a}(0):=x$ and $\lambda_{a}(1):=x^{r}$. Then we get a minimal geodesic,

$$
P_{1}:=\lambda_{a}(0) \lambda_{a}(1) .
$$

The sequence $\left\{P_{n}\right\}$ of broken geodesic segments is constructed inductively. Let $m_{k}^{n}$ denote a dyadic number $k \cdot(1 / 2)^{n}$ in $[0,1]$. For the broken geodesic segment

$$
P_{n}=\bigcup_{1 \leq k<1+2^{n-1}} \lambda_{a}\left(m_{k-1}^{n-1}\right) \lambda_{a}\left(m_{k}^{n-1}\right) \quad(n \geq 1)
$$

in $\overline{X^{a}}$ we construct the broken geodesic segment $P_{n+1}$ as follows. Let $z\left(m_{2 k-1}^{n-1}\right)$ be a middle point of the minimal geodesic $\lambda_{a}\left(m_{k-1}^{n-1}\right) \lambda_{a}\left(m_{k}^{n-1}\right)$. Because

$$
\lambda_{a}\left(m_{k-1}^{n-1}\right) \lambda_{a}\left(m_{k}^{n-1}\right) \subset \overline{X^{a}},
$$

we get a point $\lambda_{a}\left(m_{2 k-1}^{n-1}\right):=p z\left(m_{2 k-1}^{n-1}\right) \cap \partial\left(\overline{X^{a}}\right)$. The broken geodesic segment $P_{n+1}$ is defined by

$$
P_{n+1}:=\bigcup_{1 \leq k<1+2^{n}} \lambda_{a}\left(m_{k-1}^{n}\right) \lambda_{a}\left(m_{k}^{n}\right) .
$$

Letting $n \rightarrow \infty$, any rational number in $[0,1]$ corresponds to a unique point on $\partial\left(\overline{X^{a}}\right)$. Therefore such a sequence $\left\{P_{n}\right\}$ of broken geodesic segments converges to a curve $\lambda_{a}:[0,1] \longrightarrow \partial\left(\overline{X^{a}}\right)$ joining $x=\lambda_{a}(0)$ to $x^{r}=\lambda_{a}(1)$.

The same construction for the left-hand side similarly yields a certain curve $\lambda_{a}:[-1,0] \longrightarrow \partial\left(\overline{X^{a}}\right)$ joining $x^{l}=\lambda_{a}(-1)$ to $\lambda_{a}(0)=x$. From the inequality (b.1), the two curve together form the desired curve $\lambda_{a}:[-1,1] \longrightarrow \partial\left(\overline{X^{a}}\right)$ such that $\lambda_{a}(0)=x$.

To complete the proof, we now show that there is no other structure of $\partial\left(\overline{X^{a}}\right)$ around $x$ except $\lambda_{a}([-1,1])$. Take a disk neighborhood $B(x ; r)$ for a sufficiently small $r>0$ such that $B(x ; r)$ is divided into two disk domains by $\lambda_{a}([-1,1])$. Then, by the total convexity of $\operatorname{Low}(a)$, one of the components of $B(x ; r) \backslash \lambda_{a}([-1,1])$ consists of points on $\operatorname{Low}(a)$, and necessarily, the other one consists of points on $U p p(a)$. Thus the proof is complete.

Proposition 3.4. The following statements are true.

1. We have $\partial\left(\overline{X^{a}}\right) \cap \partial\left(\overline{X^{b}}\right)=\emptyset$ for every $a, b$ with $\inf _{X} \varphi<a<b<\sup _{c l} \varphi$.

2. If $x \in \partial\left(\overline{X^{a}}\right)$ for some $a \in\left(\inf _{X} \varphi, \sup _{c l} \varphi\right)$, then $x \in \operatorname{Low}(a+\varepsilon)$ for all $\varepsilon>0$.

3. $\overline{X^{a}}$ is a totally convex set with interior for every $a \in\left(\inf _{X} \varphi, \sup _{c l} \varphi\right)$. 
4. If $\inf _{X} \varphi<a<\sup _{c l} \varphi$, then for every $x \in \overline{X_{a}^{b}}$ there exists a unique number $c \in[a, b]$ such that $x \in \partial\left(\overline{X^{c}}\right)$.

5. We have for any $x \in \partial\left(\overline{X^{a}}\right)\left(a>\inf _{X} \varphi\right)$ and for any geodesic $\gamma:[a, b] \longrightarrow X$ $(\gamma(a)=x) \lim _{t \downarrow a} \varphi \circ \gamma(t)=a$.

6. If $p \in \partial\left(\overline{X^{a}}\right)$ for some $a \geq \inf _{X} \varphi$ is an interior of some geodesic $\gamma$, then $\varphi(p)=a$.

7. Let $a \in\left(\inf _{X} \varphi, \sup _{c l} \varphi\right)$. Then for given $\varepsilon>0$ and for a bounded domain $A$ with $\partial\left(\overline{X^{a}}\right) \cap A \neq \emptyset$ there exists a $\delta>0$ such that if $|t-a|<\delta$, then

$$
B\left(\partial\left(\overline{X^{a}}\right) ; \varepsilon\right) \cap A \supset \partial\left(\overline{X^{t}}\right) \cap A .
$$

Remark 3.5 (the continuous reconstruction of $\varphi$ ). It turns out from Propositions 3.4 (1)-(6) that we can reconstruct a (discontinuous) convex function $\varphi: X \longrightarrow R$ to be continuous as follows. Let $b \in\left[\inf _{X} \varphi, \sup _{c l} \varphi\right)$. We define a map $\bar{\varphi}: \overline{X^{b}} \longrightarrow R$ by

$$
\bar{\varphi}(x):= \begin{cases}c & \text { if } x \in \partial \overline{X^{c}} \text { for some } c \in\left(\inf _{X} \varphi, b\right], \\ \inf _{X} \varphi & \text { if } x \in \bigcap_{a>\inf _{X} \varphi} \operatorname{Low}(a) .\end{cases}
$$

Then $\bar{\varphi}$ is a continuous convex function on $\overline{X^{b}}$, and $\left.\bar{\varphi}\right|_{\overline{X^{b}}-J}=\left.\varphi\right|_{\overline{X^{b}}-J}$, where $J:=$ $\{$ all points at which the values of $\varphi$ are jumping up $\}$.

For a regular point $x$ in $\hat{S}_{X}$ we define

$\Sigma_{x}^{\pi}:=\left\{\xi \in \Sigma_{x} \mid\right.$ there exist geodesics $\gamma_{\xi}$ and $\gamma_{-\xi}$ tangent to $\xi$ and $-\xi$ respectively $\}$.

Then $\Sigma_{x}^{\pi}$ is of full measure in $\Sigma_{x}$. Fix a $\xi \in \Sigma_{x}^{\pi}$. Then the join $\gamma_{\xi} * \gamma_{-\xi}$ of $\gamma_{\xi}$ and $\gamma_{-\xi}$ at $x$ is not a geodesic but $\gamma_{\xi} * \gamma_{-\xi}$ behaves like a geodesic in the sense that $\bar{\varphi} \circ\left(\gamma_{\xi} * \gamma_{-\xi}\right)$ is still convex. Namely, we have $-\left(\varphi \circ \gamma_{-\xi}\right)_{+}^{\prime}(0) \leq\left(\varphi \circ \gamma_{\xi}\right)_{+}^{\prime}(0)$, where $(\cdot)_{+}^{\prime}$ is the right-hand derivative.

Proof of Proposition 3.4. (1) Suppose $\partial\left(\overline{X^{a}}\right) \cap \partial\left(\overline{X^{b}}\right) \neq \emptyset$ for some $a<b$. We use the same definitions for $x, p, w$ and $\zeta$ as in the proof of Theorem B. If such an $x$ belongs to $\partial\left(\overline{X^{a}}\right) \cap \partial\left(\overline{X^{b}}\right)$, then there exists a sequence $\left\{x_{i}\right\} \subset X^{<a}(\subset \operatorname{Low}(a))$ such that $x_{i} \rightarrow x$ as $i \rightarrow \infty$. Let $\gamma_{i}:\left[0, l_{i}\right] \longrightarrow X$ for each $i$ be a minimal geodesic joining $x_{i}$ and $p$. Then $\gamma_{i}$ intersects $\partial\left(\overline{X^{a}}\right)$ and $\partial\left(\overline{X^{b}}\right)$ at $\gamma_{i}\left(a_{i}\right)$ and $\gamma_{i}\left(b_{i}\right)$, respectively. We have from Lemma 1.1 that $\varphi \circ \gamma_{i}\left(a_{i}\right)=a$ and $\varphi \circ \gamma_{i}\left(b_{i}\right)=b$. Moreover, by the slope inequality, we have for each $i$

$$
\frac{b-a}{b_{i}-a_{i}} \leq \frac{\varphi(p)-b}{d\left(\gamma_{i}\left(b_{i}\right), p\right)}
$$

By Theorem B, it holds that $\gamma_{i}\left(a_{i}\right), \gamma_{i}\left(b_{i}\right) \rightarrow x$ as $i \rightarrow \infty$. Thus a contradiction is obtained with

$$
\liminf _{i \rightarrow \infty} \frac{b-a}{b_{i}-a_{i}}=+\infty
$$

(2) This follows from (1).

(3) This can be obtained directly from (2).

(4) We set for $x \in \overline{\operatorname{Upp}(a)} \cap \overline{\operatorname{Low}(b)}$

$$
S_{x}:=\sup \{a \mid x \in U \operatorname{Upp}(a)\}, I_{x}:=\inf \{a \mid x \in \operatorname{Low}(a)\} .
$$


Because $\operatorname{Low}(a) \cap U p p(a)=\emptyset$ for any $a \geq \inf _{X} \varphi$ and because $\operatorname{Low}(a)$ and $\operatorname{Upp}(a)$ are monotone increasing and monotone decreasing, respectively, we have $c:=S_{x}=$ $I_{x}$. Thus $x \in \partial\left(\overline{X^{c}}\right)$. The uniqueness of such a $c$ follows from Proposition 3.4 (1).

(5) This follows from (4).

(6) This follows from (1).

(7) Suppose that there exists $\varepsilon_{0}>0$ such that $B\left(\partial\left(\overline{X^{a}}\right) ; \varepsilon_{0}\right) \cap A \not \supset \partial\left(\overline{X^{a+\delta}}\right) \cap A$ (or $\partial\left(\overline{X^{a-\delta}}\right) \cap A$ ) for any $\delta>0$. Then there exist a sequence $\left\{z_{i}\right\}$ of points in $A$ and a sequence $\left\{\delta_{i}\right\}$ of positive numbers such that $z_{i} \rightarrow z \in \bar{A}$ as $i \rightarrow \infty$ and such that $z_{i}$ lies on the $\left(a+\delta_{i}\right)$-level (or $\left(a-\delta_{i}\right)$-level) and $\delta_{i} \rightarrow 0$ as $i \rightarrow \infty$. Clearly,

$$
\infty>\sup _{i} d\left(z_{i}, \partial\left(\overline{X^{a}}\right)\right) \geq \inf _{i} d\left(z_{i}, \partial\left(\overline{X^{a}}\right)\right) \geq \varepsilon_{0} .
$$

However, from Remark 3.5, we have $\lim _{i \rightarrow \infty} \bar{\varphi}\left(z_{i}\right)=\bar{\varphi}(z)=a$, where $\bar{\varphi}$ is the continuous reconstruction of $\varphi$. Hence $z \in \partial\left(\overline{X^{a}}\right)$, a contradiction.

Proof of Theorem C. Suppose that $\partial\left(\overline{X^{c}}\right)$ for some $c \in\left(a, \sup _{c l} \varphi\right)$ is noncompact. Then there exists a sequence $\left\{q_{i}\right\}$ in $\partial\left(\overline{X^{c}}\right)$ such that $\varphi\left(q_{i}\right)=c$ and $d\left(q_{i}, \partial\left(\overline{X^{a}}\right)\right) \rightarrow$ $\infty$ as $i \rightarrow \infty$.

Because $\partial\left(\overline{X^{a}}\right)$ is compact, there exists a sufficiently small positive number $\delta$ such that $\partial\left(\overline{X^{a+\delta}}\right)$ is compact. Choose a point $p \in \partial\left(\overline{X^{a}}\right)$ such that $\varphi(p)=a$ and a minimal geodesic $\sigma_{i}:\left[0, l_{i}\right] \longrightarrow X$ joining $p$ to $q_{i}$ for each $i$. Then we find a point $r_{i} \in \partial\left(\overline{X^{a+\delta}}\right) \cap \sigma_{i}$ for each $i$. It follows from the continuity of $\varphi \circ \sigma_{i}:\left(0, l_{i}\right] \longrightarrow R$ that $\varphi\left(r_{i}\right)=a+\delta$. By the slope inequality,

$$
d\left(p, r_{i}\right) \geq \frac{\delta}{c-a} \cdot d\left(p, q_{i}\right),
$$

and hence $d\left(p, r_{i}\right) \rightarrow \infty$ as $i \rightarrow \infty$, a contradiction.

Proposition 3.6. The following assertions hold.

1. The a-level set $\partial\left(\overline{X^{a}}\right)$ for every $a \in\left(\inf _{X} \varphi, \sup _{c l} \varphi\right)$ is a rectifiable curve.

2. Assume that a sequence $\left\{\mathcal{C}_{i}:[0,1] \longrightarrow \partial\left(\overline{X^{c_{i}}}\right)\right\}_{i=1}^{\infty}$ of curves in $c_{i}$-level converges pointwise to a curve $\mathcal{C}:[0,1] \longrightarrow \partial\left(\overline{X^{c_{0}}}\right)$ in the $c_{0}$-level set, where $c_{i} \in\left(\inf _{X} \varphi, \sup _{c l} \varphi\right)$ for all $i=0,1, \cdots$. Then

$$
\lim _{i \rightarrow \infty} L\left(\mathcal{C}_{i}\right)=L(\mathcal{C})
$$

Proof. (1) Let $\lambda_{a}:[0,1] \longrightarrow \partial\left(\overline{X^{a}}\right)$ be the same as in Theorem B. At each $t \in$ $(0,1)$ this $\lambda_{a}$ has the left-tangent $-\dot{\lambda}_{a}(t)$ and the right-tangent $+\dot{\lambda}_{a}(t)$. Define for $t \in(0,1)$

$$
\theta_{a}^{ \pm}(t):=\min \angle_{\lambda_{a}(t)}\left( \pm \dot{\lambda}_{a}(t), \dot{\sigma}_{\lambda_{a}(t) \lambda_{a}(0)}\right)
$$

where minimum is taken over all minimal geodesics from $\lambda_{a}(t)$ to $\lambda_{a}(0)$. We assert that

$$
\lim _{t \rightarrow 0+} \theta_{a}^{-}(t)=0
$$

In fact, the Toponogov comparison theorem and Lemma 3.2 imply the above assertion. In particular, for $x=\lambda_{a}(0) \in \partial\left(\overline{X^{a}}\right)$ and for $\delta \in(0, \pi / 2)$ there exists $\nu=\nu(x, \delta)>0$ such that

$$
\theta_{a}^{-}(t) \leq \frac{\pi}{2}-\delta
$$


for every $t \in(0, \nu]$. Thus the distance function $\rho_{a}:[0, \nu] \longrightarrow R$ defined by $\rho_{a}(t):=$ $d\left(\lambda_{a}(0), \lambda_{a}(t)\right)$ is strictly increasing in $t \in[0, \nu]$. Therefore we can reparameterize $\lambda_{a}$ by $\lambda_{a}^{\rho}:\left[0, \rho_{a}(\nu)\right] \longrightarrow \partial\left(\overline{X^{a}}\right)$ so as to satisfy $t=d\left(\lambda_{a}^{\rho}(0), \lambda_{a}^{\rho}(t)\right)$ and $\lambda_{a}^{\rho}(0)=\lambda_{a}(0)$. Then the length $L\left(\left.\lambda^{\rho}\right|_{[0, t]}\right)$ of $\left.\lambda^{\rho}\right|_{[0, t]}$ is expressed by

$$
L\left(\left.\lambda_{a}^{\rho}\right|_{[0, t]}\right)=\int_{0}^{t} \frac{d s}{\cos \theta_{a}^{-}(s)}
$$

for every $t \in\left[0, \rho_{a}(\nu)\right]$. Moreover, from (3.6.1) the above integral is finite.

(2) Fix a base line $x:\left[c_{0}-\psi, c_{0}+\psi\right] \longrightarrow X$ for a sufficiently small $\psi>0$ such that for every $t \in\left[c_{0}-\psi, c_{0}+\psi\right], x(t)$ lies on $t$-level set. We may assume $c_{i} \in\left[c_{0}-\psi, c_{0}+\psi\right]$ for all $i=0,1, \cdots$. Here $\delta \in(0, \pi / 2)$ is fixed independently of all $c_{i}$. Then, in the same manner as in the proof of Proposition 3.6 (1), we can find a positive number $\nu(i):=\nu\left(x\left(c_{i}\right), \delta\right)$ and parameterize the $c_{i}$-level set for each $i=0,1, \cdots$ by $\lambda_{c_{i}}^{\rho}:\left[0, \rho_{c_{i}}(\nu(i))\right] \longrightarrow \partial\left(\overline{X^{c_{i}}}\right)$ satisfying the conditions $\lambda_{c_{i}}^{\rho}(0)=x\left(c_{i}\right), s=d\left(\lambda_{c_{i}}^{\rho}(0), \lambda_{c_{i}}^{\rho}(s)\right)$ and $\theta_{c_{i}}^{-}(s) \leq \pi / 2-\delta$ for every $s \in\left[0, \rho_{c_{i}}(\nu(i))\right]$. The following Lemma 3.7 implies that if we put $\left.\rho:=\inf _{0 \leq i} \rho_{c_{i}}(\nu(i))\right)$, then the integrand in (3.6.2) converges to $1 / \cos \theta_{c_{0}}^{-}(s)$ almost everywhere $s \in[0, \rho]$. Thus the bounded convergence theorem implies that

$$
\lim _{i \rightarrow \infty} L\left(C_{i} \cap \overline{B\left(x\left(\left[c_{0}-\psi, c_{0}+\psi\right]\right) ; \rho\right)}\right)=L\left(C \cap \overline{B\left(x\left(\left[c_{0}-\psi, c_{0}+\psi\right]\right) ; \rho\right)}\right),
$$

that is, the validity of assertion (2) is demonstrated locally around the base line $x$. The global parameterization of each $\mathcal{C}_{i}$ with its initial point $x\left(c_{i}\right)$ is easily obtained, and $\lim _{i \rightarrow \infty} L\left(\mathcal{C}_{i}\right)=L(\mathcal{C})$ is established. We now proceed to the statement and proof of Lemma 3.7 .

Lemma 3.7. With the above notation, the following hold.

1. If we set $C_{\beta}^{a}:=\left\{x \in \partial\left(\overline{X^{a}}\right) \mid \pi-L\left(T_{x}\right) \geq \beta\right\}$ for every $a \in\left(\inf _{X} \varphi, \sup _{c l} \varphi\right)$ and every $\beta \in(0, \pi)$, then $C_{\beta}^{a} \cap K$ contains at most finitely many elements for every compact set $K$.

2. Let $\lambda_{c_{0}}^{\rho}\left(s_{0}\right)$ satisfy the equality $L\left(T_{\lambda_{c_{0}}^{\rho}\left(s_{0}\right)}\right)=\pi$ and the condition that there exists only one minimal geodesic from $\lambda_{c_{0}}^{\rho}\left(s_{0}\right)$ to $\lambda_{c_{0}}^{\rho}(0)$. If there exists a sequence $\left\{\lambda_{c_{i}}^{\rho}\left(s_{i}\right)\right\}_{i=1}^{\infty}$ of points on the $c_{i}$-level set with $c_{i} \in[a, b]$ such that $\lambda_{c_{i}}^{\rho}\left(s_{i}\right) \rightarrow \lambda_{c_{0}}^{\rho}\left(s_{0}\right)$ as $i \rightarrow \infty$, then

$$
\lim _{i \rightarrow \infty} \theta_{c_{i}}^{ \pm}\left(s_{i}\right)=\theta_{c_{0}}^{ \pm}\left(s_{0}\right)
$$

3. $\inf _{0 \leq i} \rho_{c_{i}}(\nu(i))>0$ for every $\delta \in(0, \pi / 2)$.

Proof. The first statement follows from the Toponogov comparison theorem and Lemma 3.2 .

The first step of the proof of (2) is to show that $\lim _{i \rightarrow \infty} L\left(T_{\lambda_{c_{i}}^{o}\left(s_{i}\right)}\right)=\pi=$ $L\left(T_{\lambda_{c_{0}}^{\rho}\left(s_{0}\right)}\right)$. We put $\lambda_{i}:=\lambda_{c_{i}}^{\rho}\left(s_{i}\right)(i=0,1, \cdots)$ for convenience. Since $L\left(T_{\lambda_{i}}\right) \leq \pi$ for each $i, \limsup \sup _{i \rightarrow \infty} L\left(T_{\lambda_{i}}\right) \leq \pi$. Suppose that $\liminf _{i \rightarrow \infty} L\left(T_{\lambda_{i}}\right)<\pi$. We may assume that the sequence $\left\{\lambda_{i}\right\}_{i=1}^{\infty}$ realizes the above limit-infinimum. Set

$$
\operatorname{Lim}\left(T_{\lambda_{i}}\right):=\left\{\xi \in T_{\lambda_{0}}^{\prime} \mid \text { there exists a sequence }\left\{\xi_{i}\right\} \text { with } \xi_{i} \in T_{\lambda_{i}}^{\prime}\right.
$$

$$
\text { such that } \left.\gamma_{\xi_{i}} \rightarrow \gamma_{\xi} \text { as } i \rightarrow \infty\right\}
$$


where $\gamma_{\xi_{i}}$ and $\gamma_{\xi}$ are the geodesics tangent to $\xi_{i}$ and $\xi$ respectively. Then we have

$$
\operatorname{Diam}\left(\operatorname{Lim}\left(T_{\lambda_{i}}\right)\right) \leq \liminf _{i \rightarrow \infty} L\left(T_{\lambda_{i}}\right)<\pi=L\left(T_{\lambda_{0}}\right)=\operatorname{Diam}\left(T_{\lambda_{0}}\right) .
$$

Hence we can choose a direction $v$ from $\operatorname{int}\left(T_{\lambda_{0}}\right) \backslash \operatorname{Lim}\left(T_{\lambda_{i}}\right)$. Take a point $z$ sufficiently close to $\lambda_{0}$ from the interior of the geodesic $\gamma_{v}$ tangent to $v$. Then it follows from Fact 0 that an arbitrary sequence $\left\{\lambda_{i} z\right\}$ of minimal geodesics converges to $\gamma_{v}$. Hence $\left(\dot{\lambda_{i}} z\right) \in T_{\lambda_{i}}$ for all sufficiently large $i$, where $\left(\dot{\lambda_{i}} z\right)$ is the initial tangent. Thus we obtain $v \in \operatorname{Lim}\left(T_{\lambda_{i}}\right)$, a contradiction. Therefore $\lim _{i \rightarrow \infty} L\left(T_{\lambda_{i}}\right)=\pi=L\left(T_{\lambda_{0}}\right)$.

The second step of the proof of (2) is to show $\lim _{i \rightarrow \infty} \theta_{c_{i}}^{ \pm}\left(s_{i}\right)=\theta_{c_{0}}^{ \pm}\left(s_{0}\right)$. Suppose that $\lim _{i \rightarrow \infty} \theta_{c_{i}}^{ \pm}\left(s_{i}\right) \neq \theta_{c_{0}}^{ \pm}\left(s_{0}\right)$. Then we observe that $\limsup _{i \rightarrow \infty} \theta_{c_{i}}^{-}\left(s_{i}\right)<\theta_{c_{0}}^{-}\left(s_{0}\right)$ or $\lim \sup _{i \rightarrow \infty} \theta_{c_{i}}^{+}\left(s_{i}\right)<\theta_{c_{0}}^{+}\left(s_{0}\right)$, since $\lim _{i \rightarrow \infty}\left[\theta_{c_{i}}^{+}\left(s_{i}\right)+\theta_{c_{i}}^{-}\left(s_{i}\right)\right]=\pi$. Here we suppose that $\lim \sup _{i \rightarrow \infty} \theta_{c_{i}}^{+}\left(s_{i}\right)<\theta_{c_{0}}^{+}\left(s_{0}\right)$. Then the same argument as in the first step leads us to a contradiction.

Finally, it follows from (2) that

$$
\liminf _{i \rightarrow \infty} \rho_{c_{i}}(\nu(i)) \geq \rho_{c_{0}}(\nu(0))>0,
$$

demonstrating assertion (3).

We need Lemmas 3.8-3.10 for the proof of Theorem D. Let $\partial\left(\overline{X^{a}}\right)$ be compact for some $a \in\left(\inf _{X} \varphi, \sup _{c l} \varphi\right)$. Then choose two distinct points $p_{1}$ and $p_{2}$ in $\partial\left(\overline{X^{b}}\right)$ $\left(\inf _{X} \varphi<a<b<\sup _{c l} \varphi\right)$. Let $\sigma_{1}, \sigma_{2}:[0, b-a] \longrightarrow X$ be two minimal geodesics such that $L\left(\sigma_{i}\right)=d\left(p_{i}, \partial\left(\overline{X^{a}}\right)\right)$ for $i=1,2$, parameterized by $\sigma_{i}(h) \in \partial\left(\overline{X^{a+h}}\right)$.

Lemma 3.8. With the above notation, if $l(h):=d\left(\sigma_{1}(h), \sigma_{2}(h)\right)$ and $d_{h}:=$ $d\left(\sigma_{1}(0), \sigma_{1}(h)\right)$, then

$$
\liminf _{h \downarrow 0} \frac{l(h)-l(0)}{d_{h}} \geq 0 .
$$

Proof. Suppose that there exists a constant $0<c<1$ such that

$$
\liminf _{h \downarrow 0} \frac{l(h)-l(0)}{d_{h}} \leq-c .
$$

Then there exists a strictly decreasing sequence $\left\{h_{j}\right\}$ satisfying $h_{j} \downarrow 0$ as $j \rightarrow \infty$, such that for each $j$

$$
\frac{l\left(h_{j}\right)-l(0)}{d_{h_{j}}}<-c^{2}
$$

Hence for each $j$

$$
l(0)-l\left(h_{j}\right)>c^{2} d_{h_{j}} .
$$

Let $\tau_{j}:\left[0, l\left(h_{j}\right)\right] \longrightarrow X$ be a minimal geodesic such that $\tau_{j}(0)=\sigma_{1}\left(h_{j}\right), \tau_{j}\left(l\left(h_{j}\right)\right)=$ $\sigma_{2}\left(h_{j}\right)$ for each $j$. Then the sequence $\left\{\tau_{j}\right\}$ converges to a minimal geodesic $\tau$ : $[0, l(0)] \longrightarrow X$ joining $\sigma_{1}(0)$ to $\sigma_{2}(0)$. Clearly we have

$$
\angle_{\sigma_{1}(0)}\left(\sigma_{1}, \tau\right), \angle_{\sigma_{2}(0)}\left(\sigma_{2}, \tau^{-1}\right) \geq \frac{\pi}{2},
$$

where $\tau^{-1}$ is the minimal geodesic of direction opposite to that of $\tau$. It follows from the inequality (3.8.1) that for each $j$ we can take a point $q_{j}$ on the minimal geodesic $\tau$ such that

$$
d\left(\sigma_{2}(0), q_{j}\right)=l\left(h_{j}\right)
$$


and hence

$$
d\left(\sigma_{1}(0), q_{j}\right)>c^{2} d_{h_{j}} .
$$

Because $\tau_{j} \rightarrow \tau$ as $j \rightarrow \infty$, and because $\angle_{\sigma_{2}(0)}\left(\sigma_{2}, \tau\right) \geq \frac{\pi}{2}$, there exists a sequence $\left\{\sigma_{1}\left(h_{j}\right) q_{j}\right\}$ of minimal geodesics such that

$$
\limsup _{j \rightarrow \infty} \angle \sigma_{1}\left(h_{j}\right) q_{j} \sigma_{2}(0) \leq \frac{\pi}{2} .
$$

Then from $\angle \sigma_{1}\left(h_{j}\right) q_{j} \sigma_{2}(0)+\angle \sigma_{1}\left(h_{j}\right) q_{j} \sigma_{1}(0)=\pi$ for each $j$, we have

$$
\liminf _{j \rightarrow \infty} \angle \sigma_{1}\left(h_{j}\right) q_{j} \sigma_{1}(0) \geq \frac{\pi}{2} .
$$

We see that the sequence $\left\{\triangle\left(\sigma_{1}\left(h_{j}\right) q_{j} \sigma_{1}(0)\right)\right\}$ of geodesic triangles converges to a non-degenerate flat triangle in the tangent cone $K\left(\Sigma_{\sigma_{1}(0)}\right)$ via $1 / d_{h_{j}}$-scaling. In particular we have $\angle q_{j} \sigma_{1}\left(h_{j}\right) \sigma_{1}(0)=0$. This is a contradiction.

A closed convex set $A$ in a Riemannian manifold admits a tubular neighborhood $U$ such that the distance function to $A$ is $C^{1}$-differentiable on $U \backslash A$ (see [6], Proposition 1.2). However, this is not true in our case, because the cut locus $\operatorname{Cut}\left(\partial\left(\overline{X^{a}}\right)\right)$ to $\partial\left(\overline{X^{a}}\right)$ may have a sequence of points converging to a point on $\partial\left(\overline{X^{a}}\right)$. Now, let $\Gamma(q ; a)$ for $q \in X$ and $a \in\left(\inf _{X} \varphi, \sup _{c l} \varphi\right)$ denote the set of all minimal geodesics from $q$ to $\overline{X^{a}}$. Assume that there exists for some $p$ in the $a$-level set $\left(\inf _{X} \varphi<a<\sup _{c l} \varphi\right)$ a 1-parameter family $\left\{\triangle_{h}=\triangle\left(q_{h}, r_{h}, s_{h}\right)\right\}_{h>0}$ of geodesic triangles each member of which has the vertices $q_{h}, r_{h}$ and $s_{h}$ such that

1. there exist two elements $\sigma_{1, h}, \sigma_{2, h}$ in $\Gamma\left(q_{h} ; a\right)$ such that $r_{h}=\sigma_{1, h} \cap \overline{X^{a}}, s_{h}=$ $\sigma_{2, h} \cap \overline{X^{a}}$,

2. $p \in$ int $\triangle_{h}$, and

3. $\triangle_{h} \rightarrow\{p\}$ as $h \downarrow 0$.

Set $\theta(h):=\angle_{q_{h}}\left(\sigma_{1, h}, \sigma_{2, h}\right)$. Then from Fact 6 we have

$$
\underset{h \downarrow 0}{\limsup } \theta(h) \leq 2 \pi-L\left(\Sigma_{p}\right) \leq \pi-L\left(T_{p}\right) .
$$

Thus if $p$ is a regular point, then $\lim _{h \downarrow 0} d\left(r_{h}, s_{h}\right) / d\left(q_{h}, r_{h}\right)=0$. Otherwise, if $p \in r_{h} s_{h}$ for every sufficiently small $h>0$, then $\lim _{h \downarrow 0} \theta(h)=0$, and hence $\lim _{h \downarrow 0} d\left(r_{h}, s_{h}\right) / d\left(q_{h}, r_{h}\right)=0$. When such a $p$ is singular, the following lemma holds.

Lemma 3.9. Assume that $p \in \partial\left(\overline{X^{a}}\right)$ is a singular point. If there exists a 1parameter family $\left\{\triangle_{h}\right\}_{h>0}$ of geodesic triangles satisfying (1)-(3) above, then

$$
\underset{h \downarrow 0}{\limsup } \theta(h)=0,
$$

and hence

$$
\lim _{h \downarrow 0} d\left(r_{h}, s_{h}\right) / d\left(q_{h}, r_{h}\right)=0 .
$$

Proof. It is clear that $p$ is an accumulation point of cut points to $\overline{X^{a}}$ (for details, see [14]). Hence there exists a Jordan curve $m:[0,1] \longrightarrow C u t\left(\overline{X^{a}}\right) \cup\{p\}$ such that $q_{h} \in m([0,1])$ for a sufficiently small $h>0$ and such that $p=m(0), m((0,1]) \subset$ 
$\operatorname{Cut}\left(\overline{X^{a}}\right)$. We assume $q_{h}=m(h)$. According to Lemma 2.1 in [14], $m$ has the right-tangent $+\dot{m}(0)$ at $p=m(0)$ and

$$
\lim _{h \downarrow 0}(p \dot{m}(h))=+\dot{m}(0) .
$$

Then we have $+\dot{m}(0) \notin \operatorname{int}\left(T_{p}\right)$. Letting $\left\{v_{p}^{r}, v_{p}^{l}\right\}=\partial T_{p}$, we have from Lemma 3.2

$$
\angle\left(+\dot{m}(0), v_{p}^{r}\right) \geq \frac{\pi}{2} \text { or } \angle\left(+\dot{m}(0), v_{p}^{l}\right) \geq \frac{\pi}{2} .
$$

For $i=1,2$ and $h>0$, let $\xi_{i, h}$ be a minimal geodesic joining $\sigma_{i, h} \cap \overline{X^{a}}$ to $p$ and $\triangle_{i, h}$ a geodesic triangle surrounded by $\sigma_{i, h}, p m(h)$ and $\xi_{i, h}$. Here we suppose that $\angle_{m(h)}\left(\sigma_{1, h}, \sigma_{2, h}\right)=\max \left\{\angle_{m(h)}(\gamma, \sigma) \mid \gamma, \sigma \in \Gamma(m(h) ; a)\right\}$ and that $\lim _{h \downarrow 0} \dot{\xi}_{1, h}=v_{p}^{l}$, $\lim _{h \downarrow 0} \dot{\xi}_{2, h}=v_{p}^{r}$. Suppose that

$$
\angle_{o_{p}}\left(+\dot{m}(0), v_{p}^{l}\right)=\lim _{h \downarrow 0} \angle_{p}\left((p m(h)), \dot{\xi}_{1, h}\right) \geq \pi / 2 .
$$

Then we conclude from Fact 5 that $\triangle_{1, h}$ tends to a flat triangle on $K\left(\Sigma_{p}\right)$ by the $1 / d(m(h), p)$-scaling as $h \rightarrow 0$. Thus we have

$$
\lim _{h \downarrow 0} \angle_{p}\left(+\dot{m}(0), \dot{\xi}_{1, h}\right)=\angle_{p}\left(+\dot{m}(0), v_{p}^{l}\right)=\pi / 2 .
$$

By the same reasoning, we have $\angle_{p}\left(+\dot{m}(0), v_{p}^{r}\right)=\pi / 2$. Therefore $\lim \sup _{h \downarrow 0} \theta(h) \leq$ $\lim _{h \downarrow 0} \angle\left(\sigma_{1, h}, \sigma_{2, h}\right)=0$.

With the same notation as in the proof of Theorem B, we prove

Lemma 3.10. Let $x=\lambda_{a}(0) \in \partial\left(\overline{X^{a}}\right)$ satisfy $L\left(T_{x}\right)>\pi-\delta$ for a small $\delta>0$. Then

$$
1 \leq \liminf _{s \rightarrow 0-, t \rightarrow 0+} \frac{L\left(\left.\lambda_{a}\right|_{[s, t]}\right)}{d\left(\lambda_{a}(s), \lambda_{a}(t)\right)} \leq \limsup _{s \rightarrow 0-, t \rightarrow 0+} \frac{L\left(\left.\lambda_{a}\right|_{[s, t]}\right)}{d\left(\lambda_{a}(s), \lambda_{a}(t)\right)} \leq \frac{1}{\cos (\delta / 2)} .
$$

Proof. It suffices to prove limsup $\sup _{s \rightarrow 0-, t \rightarrow 0+} L\left(\left.\lambda_{a}\right|_{[s, t]}\right) / d\left(\lambda_{a}(s), \lambda_{a}(t)\right) \leq 1 / \cos (\delta / 2)$. By taking a suitable scaling, we see that a region which is surrounded by $\lambda_{a}([s, t])$ and a minimal geodesic from $\lambda_{a}(s)$ to $\lambda_{a}(t)$ tends to a triangle $\triangle$ on $\left(K\left(\Sigma_{x}\right), o_{x}\right)$ such that the angle at $o_{x}$ is greater than $\pi-\delta$ and such that the edge opposite to $o_{x}$ is 1 . Then

$$
\limsup _{s \rightarrow 0-, t \rightarrow 0+} \frac{L\left(\left.\lambda_{a}\right|_{[s, t]}\right)}{d\left(\lambda_{a}(s), \lambda_{a}(t)\right)} \leq \frac{1}{\cos (\delta / 2)} .
$$

Proof of Theorem D. We prove that for every $b \in\left[a, \sup _{c l} \varphi\right)$

$$
\liminf _{h \downarrow 0} \frac{L\left(\partial\left(\overline{X^{b+h}}\right)\right)-L\left(\partial\left(\overline{X^{b}}\right)\right)}{M_{h}} \geq 0,
$$

where $M_{h}:=\max \left\{d\left(x, \partial\left(\overline{X^{b}}\right)\right) \mid x \in \partial\left(\overline{X^{b+h}}\right)\right\}$.

For any $h>0$ with $b+h<\sup _{c l} \varphi$ let $\pi^{h}: \partial\left(\overline{X^{b+h}}\right) \longrightarrow \overline{X^{b}}$ satisfy $d\left(x, \pi^{h}(x)\right)=$ $d\left(x, \overline{X^{b}}\right)$. From Proposition $3.4(7)$ we conclude that there exist for every $h>0$ an integer $N_{h}$ and a positive $\varepsilon_{h}$ such that

$$
L\left(\partial\left(\overline{X^{b}}\right)\right)-\sum_{j=1}^{N_{h}} d\left(\pi^{h}\left(x_{j}^{h}\right), \pi^{h}\left(x_{j+1}^{h}\right)\right)<\varepsilon_{h},
$$


where $\left\{x_{j}^{h}\right\}$ is a partition of $\partial\left(\overline{X^{b+h}}\right)$ such that $N_{h} \rightarrow \infty, \varepsilon_{h} \rightarrow 0$ as $h \downarrow 0$. Put

$$
c_{j, h}:=\frac{d\left(x_{j}^{h}, x_{j+1}^{h}\right)-d\left(\pi^{h}\left(x_{j}^{h}\right), \pi^{h}\left(x_{j+1}^{h}\right)\right)}{M_{h}} .
$$

Then the inequality $\lim \inf _{h \downarrow 0} c_{j, h} \geq 0$ for each $j$ follows from Lemma 3.8. Therefore

$$
\begin{gathered}
\liminf _{h \downarrow 0} \frac{L(b+h)-L(b)}{M_{h}} \geq \liminf _{h \downarrow 0} \sum_{j=1}^{N_{h}} c_{j, h}-\underset{h \downarrow 0}{\limsup } \frac{\varepsilon_{h}}{M_{h}} \\
\geq \sum_{j=1}^{\infty} \liminf _{h \downarrow 0} c_{j, h}-\underset{h \downarrow 0}{\limsup } \frac{\varepsilon_{h}}{M_{h}} \geq-\underset{h \downarrow 0}{\limsup } \frac{\varepsilon_{h}}{M_{h}} .
\end{gathered}
$$

Lemmas 3.9 and 3.10 imply that $\lim \sup _{h \downarrow 0} \varepsilon_{h} / M_{h}=0$. This proves the monotone property of $L$. The continuity of $L$ follows from Proposition 3.6.

Proof of corollary to $B, C$ and $D$. (1) Suppose that there is a noncompact level $\partial\left(\overline{X^{b}}\right)$ with $b \in\left(\inf _{X} \varphi, \sup _{c l} \varphi\right)$. Then $b<a$ follows from Theorem C. Define

$$
b_{0}:=\sup \left\{b \mid \partial\left(\overline{X^{b}}\right) \text { is noncompact }\right\} .
$$

Choose a strictly decreasing sequence $\left\{\varepsilon_{j}\right\}$ such that $\varepsilon_{j} \downarrow 0$ as $j \rightarrow \infty$. Let $\left\{C_{1}^{j}, \cdots, C_{n(j)}^{j}\right\}$ be the collection of components of $\partial\left(\overline{X^{b_{0}+\varepsilon_{j}}}\right)$, where $n(j)$ is the number of components of $\partial\left(\overline{X^{b_{0}+\varepsilon_{j}}}\right)$. Then, by the convexity of $\varphi$, we have $n(j+1) \leq n(j)$ for all $j$. Thus

$$
\max _{1 \leq i \leq n(j)}\left(\text { diameter of } C_{i}^{j}\right) \rightarrow \infty \text { as } j \rightarrow \infty
$$

Also,

$$
\max _{1 \leq i \leq n(j)}\left(\text { length of } C_{i}^{j}\right) \geq \max _{1 \leq i \leq n(j)} 2\left(\text { diameter of } C_{i}^{j}\right)
$$

for each $j$, which is a contradiction of the monotone non-decreasing property of the length of levels.

(2) Suppose $\mu:=\sup _{c l} \varphi<\infty$. Fix a point $p_{0}$ on the $c_{0}$-level set with $c_{0} \in$ (inf $\left.{ }_{X} \varphi, \mu\right)$. Then our claim is the following.

Claim. There exists a broken geodesic segment $\gamma$ emanating from $p_{0}$ such that $\gamma$ intersects the $t$-level sets for all $t \in\left[c_{0}, \mu\right)$ and such that the right-hand derivative $(\varphi \circ \gamma)_{+}^{\prime}$ at every point on $\gamma$ is bounded from below by a positive constant.

Assume that the above claim is true. Then $\gamma$ reaches $\bigcap_{\inf _{X} \varphi<a<\mu} U p p(a)$ after traversing a finite length. Here we choose a strictly increasing sequence $\left\{c_{i}\right\}_{i=0}^{\infty}$ such that $c_{i} \geq c_{0}(\forall i=0,1, \cdots)$ and $\lim _{i \rightarrow \infty} c_{i}=\mu$. Then the Ascoli-Arzelà theorem implies that a subsequence $\left\{\partial\left(\overline{X^{c_{i(j)}}}\right)\right\}_{j=0}^{\infty}$ of a sequence of $c_{i}$-level sets intersecting $\gamma$ converges to a curve $\mathcal{C}$ lying on $\bigcap_{\inf _{X} \varphi<a<\mu} U p p(a)$. Moreover, by Theorems $\mathrm{C}$ and $\mathrm{D}$, the length of $\partial\left(\overline{X^{c_{i(j)}}}\right)$ is non-decreasing when $j$ goes to infinity. Hence $\mathcal{C}$ is a non-trivial curve. Then take a point $r \in \mathcal{C}$ and a small disk neighborhood $U_{r}$ around $r$ such that $U_{r}$ is divided by $\mathcal{C}$ into two disjoint disk domains $U_{r}^{1}$ and $U_{r}^{2}$. From the definition of $\sup _{c l} \varphi$, we observe that $\bigcap_{\inf _{X} \varphi<a<\mu} U p p(a)$ has no interior. Thus for every small $\varepsilon>0$ we can find the $(\mu-\varepsilon)$-level sets close to $r$ in $U_{r}^{1}$ and $U_{r}^{2}$, respectively. Since $\overline{X^{\mu-\varepsilon}}$ is totally convex, this is a contradiction.

We now prove our claim. Here we prove a general property on the directional derivative $u(\varphi)$ at $p \in X$ for $u \in \Sigma_{p}^{\prime}$. We define $u(\varphi):=\left(\varphi \circ \gamma_{u}\right)_{+}^{\prime}(0)$, where 
$\gamma_{u}:\left[0, l_{u}\right] \longrightarrow X$ is a geodesic from $p$ tangent to $u$. Let $u, v$ be two directions in $\Sigma_{p}^{\prime}$ with $0<\angle(u, v)<\operatorname{diam}\left(\Sigma_{p}\right)$. Then $u$ and $v$ determine a flat triangle $\triangle\left(o_{p} ; u, v\right)$ on $K\left(\Sigma_{p}\right)$ whose edges $u$ and $v$ make an angle $\angle(u, v)$ at $o_{p}$ and both are of length 1. Let $w_{\lambda} \in \Sigma_{p}^{\prime}$ satisfy the condition that a line $l_{w_{\lambda}}$ corresponding to $w_{\lambda}$ divides the edge of $\triangle\left(o_{p} ; u, v\right)$ opposite to $o_{p}$ by the ratio $\lambda:(1-\lambda)$ for $0<\lambda \leq 1$. Then

$$
w_{\lambda}(\varphi) \leq[(1-\lambda) u(\varphi)+\lambda v(\varphi)] \cdot \frac{\sin \angle\left(u, w_{\lambda}\right)}{\lambda \sin \angle(u, v)} .
$$

From (b,c,d.1) we observe that the directional derivative $\cdot(\varphi): \Sigma_{p}^{\prime} \longrightarrow R(u \mapsto$ $u(\varphi))$ has a continuous extension, $\cdot(\varphi): \Sigma_{p} \longrightarrow R$. Moreover, for every $p \in X$ and $u \in \Sigma_{p}$ there exists $w \in \Sigma_{p}$ such that

$$
w(\varphi) \geq|u(\varphi)| .
$$

Let $u_{0} \in \Sigma_{p_{0}}^{\prime}$ satisfy $u_{0}(\varphi) \geq \delta>0$ and $\gamma_{u_{0}}:\left[0, l_{0}\right] \longrightarrow X$ be a maximal geodesic tangent to $u_{0}$. Then, by the convexity of $\varphi$, we have $\left|\left(\varphi \circ \gamma_{u_{0}}\right)_{-}^{\prime}\left(l_{0}\right)\right| \geq u_{0}(\varphi) \geq \delta$. If $\gamma_{u_{0}}\left(l_{0}\right)$ lies on the $c_{1}$-level set with $c_{1}<\mu$, then using (b,c,d.2) and the continuity of the directional derivative we can find $u_{1} \in \Sigma_{\gamma_{u_{0}}\left(l_{0}\right)}^{\prime}$ such that $u_{1}(\varphi)>\eta \cdot \delta$ for $\eta \in(0,1)$. If an endpoint $\gamma_{u_{1}}\left(l_{1}\right)$ of a maximal geodesic $\gamma_{u_{1}}:\left[0, l_{1}\right] \longrightarrow X$ tangent to $u_{1}$ is not in $\bigcap_{\inf _{X} \varphi<a<\mu} \operatorname{Upp}(a)$, then we can find $u_{2} \in \Sigma_{\gamma_{u_{1}}\left(l_{1}\right)}$ with $u_{2}(\varphi)>\eta \cdot \delta$ in the same manner as $u_{1}$. Repeating the same procedure, we obtain a broken geodesic segment $\gamma:=\bigcup_{i} \gamma_{u_{i}}$ emanating from $p_{0}$ such that $(\varphi \circ \gamma)_{+}^{\prime}>\eta \cdot \delta$ at all point on $\gamma$.

It may happen that an endpoint $q_{0}$ of $\gamma$ other than $p_{0}$ is not in $\bigcap_{\inf _{X} \varphi<a<\mu} U p p(a)$. Because $d\left(p_{0}, q_{0}\right)$ is no greater than the length of $\gamma$, we find a direction $v_{-1}$ tangent to a minimal geodesic $p_{0} q_{0}$ at an interior point of $p_{0} q_{0}$ such that $v_{-1}(\varphi)>\eta \cdot \delta$. It follows from convexity that $\left|\left(\varphi \circ\left(p_{0} q_{0}\right)\right)_{-}^{\prime}\right|$ at $q_{0}$ is greater than $\eta \cdot \delta$. Therefore, from (b,c,d.2), we can find $v_{0} \in \Sigma_{q_{0}}^{\prime}$ with $v_{0}(\varphi)>\eta \cdot \delta$, and we proceed as with the $u_{i}$. Therefore we see that $q_{0} \in \bigcap_{\inf _{X} \varphi<a<\mu} \operatorname{Upp}(a)$. This completes the proof.

(3) Suppose that $X$ is compact. Because $X=\bigcup_{a>\inf _{X} \varphi} \operatorname{Low}(a)$ and because $\operatorname{Low}(a)$ is monotone increasing, there exists a number $a_{0}$ such that $X=\operatorname{Low}\left(a_{0}\right)$. Therefore $X=\overline{X^{a_{0}}}$. Hence $\sup _{c l} \varphi<+\infty$, and this contradicts (2).

Proof of Theorem A. From the corollary to Theorems B, C and D, we have $\inf _{X} \varphi=$ $\sup _{c l} \varphi$, and hence $\overline{X^{\inf _{X} \varphi}}=X$. On the other hand, from Lemma 1.1, $\varphi(x)=$ $\inf _{X} \varphi$ follows for every $x \notin \hat{S}_{X}$.

\section{Ends AND topological STRUCTURE}

In this section, we discuss a convex function $\varphi: X \longrightarrow R$ with the property

$$
\bigcap_{a>\inf _{X} \varphi} \operatorname{Low}(a) \text { has no interior. }
$$

Clearly, (***) implies that $\inf _{X} \varphi<\sup _{c l} \varphi$. First, we prove Theorem E. Then we obtain the corollary to Theorem E, as mentioned in the Introduction. To prove Theorem E, we begin with a discussion of a homotopy deformation of $\partial\left(\overline{X^{a}}\right)$.

Assume that $\partial\left(\overline{X^{a}}\right)\left(a>\inf _{X} \varphi\right)$ contains a compact component $C$. Choose points $p_{0}, \cdots, p_{m}=p_{0}$ on $C$ such that $(1) \varphi\left(p_{i}\right)=a$ for all $i=0, \cdots, m-1$, (2) $p_{i} p_{i \pm 1}$ are contained entirely in the disk neighborhood $U_{i}$ around $p_{i}$, and (3) $C \subset \bigcup_{i=1}^{m-1} U_{i}$. Clearly $\varphi(C) \leq a$, and $C_{0}:=p_{0} p_{1} \cup \cdots \cup p_{m-1} p_{m}$ bounds a convex domain $D_{0}$ which contains sublevels below $a$. If $C_{0}$ is not a closed geodesic, then 
we apply the short-cut principle in a disk neighborhood at each corner of $C_{0}$ whose angle is less than $\pi$. We then obtain by induction a sequence of decreasing convex domains $\left\{D_{N}\right\}$ with boundaries $C_{N}$ such that $C_{N}$ for each $N$ is freely homotopic to $C$ in $\overline{X^{a}}$ and $L\left(C_{N}\right)$ is decreasing in $N$. Let $C^{\prime} \subset \partial\left(\overline{X^{a}}\right)$ be a component of $\partial\left(\overline{X^{a}}\right) \backslash C$ and fix points $p \in C, p^{\prime} \in C^{\prime}$. Then we see that $p p^{\prime} \cap C_{N} \neq \emptyset$ for all $N$. In particular, $C_{N}$ for every $N$ is contained in the ball centered at $p$ with the radius $d\left(p, p^{\prime}\right)+L(C)$. Thus the Ascoli-Arzelà theorem implies that $\left\{C_{N}\right\}_{N}$ converges to a simple closed geodesic $C_{\infty}$. Clearly $\varphi\left(C_{\infty}\right)$ is a constant.

With the above notation we prove

Lemma 4.1. There exists a sequence of simple closed geodesics converging to the minimum set of $\varphi$. Moreover, the limit of this sequence is a simple closed geodesic.

Proof. Suppose $\varphi\left(C_{\infty}\right)=: b>\inf _{X} \varphi$. Then there is a point $z \in X$ with $\varphi(z) \in$ $\left(\inf _{X} \varphi, b\right)$. Let $z y$ be a minimal geodesic from $z$ to $C_{\infty}$ such that $y \in C_{\infty}$. Then, applying the short-cut principle to a closed curve $z y \cup C_{\infty} \cup y z$ fixing the point $z$, we obtain a geodesic loop $\gamma_{z}$ at $z$ such that $\varphi\left(\gamma_{z}\right) \leq b$. Therefore the angle at $z$ of $\gamma_{z}$ is not greater than $\pi$. If the angle is less than $\pi$, we obtain a simple closed geodesic by the same procedure. Thus a sequence $\left\{C_{N}\right\}$ of simple closed geodesics is obtained in such a way that $\varphi\left(C_{N}\right)=b_{N}$ is constant and $\left\{b_{N}\right\}$ is decreasing with $\lim _{N \rightarrow \infty} b_{N}=\inf _{X} \varphi$. Lemma 1.2 then implies that

$$
C_{\infty}:=\lim _{N \rightarrow \infty} C_{N}=X^{\min _{X} \varphi}=\bigcap_{a>\inf _{X} \varphi} \operatorname{Low}(a) .
$$

Proof of Theorem E. (1) If $\partial\left(\overline{X^{a}}\right)$ has a compact component, then the infinimum of $\varphi$ is attained, by Lemma 4.1 .

If $\partial\left(\overline{X^{a}}\right)$ is noncompact, it follows from Lemma 4.1 that all the components of $\partial\left(\overline{X^{a}}\right)$ are noncompact. We choose two components $E_{1}$ and $E_{2}$ of $\partial\left(\overline{X^{a}}\right)$ and take two points $p_{1}$ and $p_{2}$ from $E_{1}$ and $E_{2}$, respectively. Suppose that there exists a geodesic $\gamma$ joining $p_{1}$ to $p_{2}$ such that $\inf _{x \in \gamma} \varphi(x)>\inf _{X} \varphi$. Then let $r \in \gamma$ be a point with $\varphi(r)=\inf _{x \in \gamma} \varphi(x)$. Clearly, $r \neq p_{1}, p_{2}$ and $\varphi(r)<a$. Note that $E_{1}(i=1,2)$ is divided by $p_{1}$ into two half lines, $E_{1}(+)$ and $E_{1}(-)$. We take an open set $U:=\bigcup_{x \in p_{1} p_{2}} U_{x}$, where $U_{x}$ is a small disk neighborhood around $x$. Put $S:=U \cap \overline{X^{a}}$. Then $S$ is a strip, and $S \backslash p_{1} p_{2}$ has two components $S(+), S(-)$. Here we assume that $E_{1}(+)$ bounds $S(+)$.

Take a point $s \in S$ such that $\varphi(s)<\varphi(r)$. Without loss of generality we may assume $s \in S(-)$. Then every geodesic from $s$ to every point of $E(+)$ meets $\gamma$, because $S$ is a strip. $E_{1}(+)$ is a proper embedding of $[0, \infty)$. Thus there exists a ray $\xi:[0, \infty) \longrightarrow X$ emanating from $s$ to the infinity of $E_{1}(+)$ such that $\xi \subset \overline{X^{a}}$ and $\gamma$ intersects $\xi$ at a point $u$. Then by the convexity of $\varphi$, we have $\lim _{t \rightarrow \infty} \varphi(\xi(t))=\infty$, a contradiction.

(2) If $\partial\left(\overline{X^{a}}\right)$ is compact, then this assertion follows from Lemma 4.1. Thus suppose that $\partial\left(\overline{X^{a}}\right)$ is noncompact. We use the same notation as in the proof of (1). Let $p$ be a point in the minimum set of $\varphi$. Since $E_{1}(+)$ and $E_{1}(-)$ are both proper embeddings of $[0, \infty)$, we can construct two rays $\gamma_{+}, \gamma_{-}:[0, \infty) \longrightarrow X\left(\gamma_{ \pm}(0)=p\right)$ associated with $E_{1}(+)$ and $E_{1}(-)$. Then we have $\gamma_{ \pm} \subset X^{\min _{X} \varphi}$ and $\gamma_{+} \cap \gamma_{-}=\{p\}$. Hence we conclude from Lemma 1.2 that $X^{\min _{X} \varphi}$ is a straight line.

(3) It follows from previous (2) that every small neighborhood $N$ of $X^{\lambda}$ is divided by $X^{\lambda}$ into exactly two components $N_{1}$ and $N_{2}$. Suppose that the $b$-level set has more than two components, let us suppose $E_{1}, E_{2}$ and $E_{3}$, for some $b \in\left(\inf _{X} \varphi, \infty\right)$. 
Take points $p_{1} \in E_{1}, p_{2} \in E_{2}$ and $p_{3} \in E_{3}$. Then from (2) we observe that every geodesic $\gamma_{i j}:\left[0, l_{i j}\right] \longrightarrow X$ from $p_{i}$ to $p_{j}\left(p_{i}=\gamma_{i j}(0)\right)$ meets $X^{\lambda}$ at a unique point $p_{i j}:=\gamma\left(s_{i j}\right)$ for $i<j$. By the convexity of $\varphi$, we see that $\gamma_{i j}$ meets both $N_{1}$ and $N_{2}$ only once. Here we assume that $\left.\gamma_{12}\right|_{\left[0, s_{12}\right]}$ meets $N_{1}$. Then $\left.\gamma_{13}\right|_{\left[0, s_{13}\right]}$ also meets $N_{1}$. If we suppose that $\left.\gamma_{13}\right|_{\left[0, s_{13}\right]}$ meets $N_{2}$, then by the short-cut principle we see that the broken geodesic polygon

$$
\left.\left.\gamma_{12}\right|_{\left[0, s_{12}\right]} \cup p_{12} p_{13} \cup \gamma_{13}\right|_{\left[0, s_{13}\right]}
$$

is deformed homotopically to a closed geodesic passing through $X^{\lambda}$. This is a contradiction of (2). By counting the intersection number of $\gamma_{23}$ and $N_{1}$ (or $N_{2}$ ), we see that $\gamma_{23}$ does not meet $N_{1}$ and hence $\gamma_{23}$ must touch $X^{\lambda}$ at $p_{23}$, contradicting Fact 0 . Thus the $b$-level set has at most two components for every $b \in\left(\inf _{X} \varphi, \infty\right)$. Moreover, from the above discussion of the intersection number, we conclude that the $b$-level set has exactly two components for every $b \in\left(\inf _{X} \varphi, a\right]$.

It remains to prove that the $b$-level set lying upon the $a$-level set has exactly two components. This follows from the fact that the number of components of the $b$-level set is nondecreasing in $b \in\left(\inf _{X} \varphi, \infty\right)$.

In the last part of this section, to prove our main theorem, the following proposition is needed.

Proposition 4.2. For every $b>\inf _{X} \varphi$ there exists a homeomorphism

$$
H: \partial\left(\overline{X^{b}}\right) \times\left(\inf _{X} \varphi,+\infty\right) \longrightarrow X-\bigcap_{a>\inf _{X} \varphi} \operatorname{Low}(a)
$$

satisfying the condition that $H(x, t) \in \partial\left(\overline{X^{t}}\right)$ for every $(x, t) \in \partial\left(\overline{X^{b}}\right) \times\left(\inf _{X} \varphi,+\infty\right)$.

Proof. We use the same notation here as in Proposition 3.6. Assume that $\varphi$ has connected, noncompact levels. Then take a base line $x:\left(\inf _{X} \varphi, \infty\right) \longrightarrow X$. If $\varphi$ has disconnected levels, then we take two such base lines. Since the $t$-level set is a rectifiable curve for every $t \in\left(\inf _{X} \varphi, \infty\right)$, there is a length parameterization $\lambda_{t}^{l}:(-\infty,+\infty) \longrightarrow \partial\left(\overline{X^{t}}\right)$ for each $t \in\left(\inf _{X} \varphi, \infty\right)$. That is, it holds that $\lambda_{t}^{l}(0)=$ $y_{0}(t)$ and $L\left(\left.\lambda_{t}^{l}\right|_{[0, s]}\right)$ (or $\left.L\left(\left.\lambda_{t}^{l}\right|_{[s, 0]}\right)\right)=|s|$. We identify $(-\infty,+\infty)$ and the $t$-level set using this $\lambda_{t}^{l}$. Then $\lambda_{t}^{l}$ gives the required homeomorphism $H$. The continuities of $H$ and $H^{-1}$ are implied by Proposition 3.6 (2).

On the other hand, when $\varphi$ has compact levels, we parameterize the $t$-level sets in such a way that the parameterization of each such set is proportional to the length of that level set for each $t \in\left(\inf _{X} \varphi, \infty\right)$.

Our Main Theorem is now obtained immediately by Proposition 4.2.

\section{REFERENCES}

1. A.D.Alexandrov, Über eine Verallgemeinerung der Riemannshen Geometrie, Schriftenreiche der Institut für Mathematik (1957), 33-84.

2. Bangert, V., Totally convex sets in complete Riemannian manifolds, J. Diff. Geom. 16 (1981), 333-345. MR 83e:53041

3. Y. Burago, M. Gromov, and G. Perelman, A. D. Alexandrov's spaces with curvatures bounded below, Russian math. surveys 47:2 (1992), 1-58. MR 93m:53035

4. H.Busemann, The Geometry of Geodesics, Academic Press, New York-London (1955). MR $17: 779 \mathrm{a}$

5. J. Cheeger and D. Gromoll, On the structure of complete manifolds of nonnegative curvature, Ann. of Math. 96 (1972), 413-443. MR 46:8121 
6. R. E. Greene and K. Shiohama, Convex functions on complete noncompact manifolds: Topological structure, Invent. Math. 63 (1981), 129-157. MR 82e:53065

7. R. E. Greene and K. Shiohama, Convex functions on complete noncompact manifolds: Differential structure, Ann. Sci. École Norm. Sup. (4),14 (1981), 357-367. MR 83m:53057

8. R. E. Greene and K. Shiohama, The isometry groups of manifolds admitting nonconstant convex functions, J. Math. Soc. Japan 39 (No.1) (1987). MR 88a:53032

9. N. Innami, A classification of Busemann G-surfaces which possess convex functions, Acta Math. 148 (1982), 15-29. MR 84f:53063

10. Y. Machigashira, The Gaussian curvature of Alexandrov surfaces, to appear.

11. Y. Otsu and T. Shioya, The Riemannian structure of Alexandrov spaces, J. Diff. Geom. 39 (1994), 629-658. MR 95e:53062

12. G. Perelman, Alexandrov's spaces with curvatures bounded from below II, preprint.

13. K. Shiohama, An Introduction to the Geometry of Alexandrov Spaces, Seoul National univ. Lecture notes series 8. MR 96c:53064

14. K. Shiohama and M. Tanaka, Cut loci and Distance spheres on Alexandrov surfaces, Proc. Round Table in Diff. Goem.-in honor of Marcel Berger-Collection SMF Séminaires \& Congrès no.1 (1996), 531-560. MR 98a:53062

Graduate School of Mathematics, Kyushu University, 6-10-1 Hakozaki, Higashi-ku, FUKUOKA, 812-81 JAPAN

E-mail address: mashiko@math.kyushu-u.ac.jp 